\title{
Average Throughput in AWGN Cognitive Fading Interference Channel with Multiple Secondary Pairs
}

\author{
Nicolas Schrammar, Hamed Farhadi, Lars K. Rasmussen, and Mikael Skoglund \\ Communication Theory Laboratory, School of Electrical Engineering \\ KTH Royal Institute of Technology, Stockholm, Sweden
}

\begin{abstract}
We consider the additive white Gaussian noise (AWGN) cognitive fading interference channel consisting of one primary and multiple secondary transmitter-receiver pairs. The secondary transmitters have non-causal knowledge of the primary message. We find a tuple of achievable rates by utilizing the discrete superposition model (DSM), which is a simplified, deterministic channel model. The coding scheme devised for the DSM can be translated into a coding scheme for the AWGN model, where the rate achieved in the AWGN model is at most a constant gap below the rate achieved in the DSM. We then calculate the average throughput of the secondary pairs under the assumption of Rayleigh fading channels. The main result is that our scheme performs well in the weak interference regime. The sum-throughput increases with the number of secondary pairs.
\end{abstract}

\section{INTRODUCTION}

The paradigm of cognitive radio [1] promises increased bandwidth efficiency by improved utilization of sparsely used spectrum. One important model for cognitive radio is the cognitive interference channel (CIFC), which consists of a primary and a secondary transmitter-receiver pair. Each transmitter sends a message to its desired receiver, which causes interference at the other receiver. Cognition is modeled by the fact that the secondary transmitter knows the primary message and, hence, the primary transmit signal non-causally. The first study of the CIFC from an information-theoretic point-of-view was [2], however, the capacity region is known only for special cases. These are weak interference [3], very strong interference [4] and "primary decodes cognitive" [5]. Several achievable rate regions have been found, see [2], [6] and references therein.

In many practical applications there exist multiple cognitive systems that wish to share the spectrum. It is an interesting question, if the simultaneous operation of the secondary systems is possible and beneficial compared to serving only one secondary system at a time. A suitable model for simultaneous operation is the $K$-user cognitive interference channel ( $K$-CIFC) consisting of one primary and $K-1$ secondary transmitter-receiver pairs. All secondary transmitters are assumed to know the primary message. Limited results are known about the capacity region of the $K$-CIFC. In [7] the

The research leading to these results has received funding from the European Union's Seventh Framework Programme FP7/2007-2013 under grant agreement $\mathrm{n}^{\circ} 248303$. The authors would like to acknowledge the contributions of their colleagues to the project.

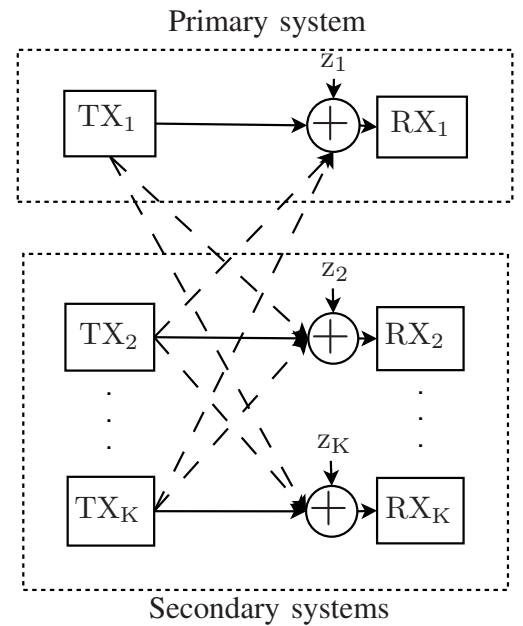

Fig. 1. Cognitive $K$-user interference channel consisting of one primary and $K-1$ secondary transmitter-receiver pairs.

capacity region for a symmetric $K$-CIFC with reduced number of cross-links is found. In [8] the authors consider a three-user scenario. References to additional results with less relation to our work can be found in [9].

In this paper we explore a novel way of finding achievable rates for the $K$-CIFC. We consider the simplified deterministic discrete superposition model (DSM) [10]. It has been shown that a code in the DSM of a $K$-user interference channel can be translated into a code in the corresponding AWGN model. The achievable rates in the AWGN model are at most a constant gap below the rates in the DSM. This correspondence holds for the $K$-CIFC as well.

We devise a specific code in the DSM based on interference avoidance and dirty paper coding (DPC) [11], and we find lower bounds on the achievable rates. Due to the above mentioned properties of the DSM, this yields lower bounds on the rates achievable in the AWGN model. Based on these results we calculate the average throughput for the case of Rayleigh fading channel gains. We compare our analytical expressions to numerical simulations, and we explore the dependency of the throughput on the number $K$ of transmitterreceiver pairs, the signal-to-noise ratio (SNR) and the strength of interference. Our results shows that average sum throughput of the secondary users increase linearly as the number of users increases which shows that this network is not interference limited in general. 
The remainder of the paper is organized as follows. In Sec. II we define the AWGN model and the DSM of the $K$ CIFC, and we discuss the connection between the two models. In Sec. III and Sec. IV we state our results, the achievable rate tuple and the average secondary throughput, respectively. Sec. V contains numerical evaluations, and Sec. VI concludes the paper.

\section{SySTEM MODEL}

We consider a single-antenna $K$-CIFC represented in Fig. 1. Each transmitter $\mathrm{TX}_{k}$ has independent messages for its dedicated receiver $\mathrm{RX}_{k}$. Since all transmitters share the transmission medium, each of the receivers gets the desired message from the corresponding transmitter over the desired channel and also receives interference from all other transmitters over interference channels. The channel is cognitive in the sense that the secondary transmitters $\mathrm{TX}_{k}, k \in\{2, \ldots, K\}$ know the message of the primary transmitter $\mathrm{TX}_{1}$.

\section{A. AWGN Model}

The AWGN model of the $K$-CIFC is

$$
y_{k}=\sum_{l=1}^{K} h_{l k} x_{l}+z_{k},
$$

where $z_{k} \sim \mathcal{C N}(0,1)$ is additive white Gaussian noise. Each transmitter has unit transmit power $\mathrm{E}\left|x_{k}\right|^{2}=1$, hence the model is completely described by the complex-valued channel gains $h_{l k}$. We assume that the channels are discrete-time block fading channels, and that the channel gains change independently. We assume global knowledge of the channel states. For simplicity, consider that all interference channel gains $h_{l k}, l \neq k$ between transmitter $\mathrm{TX}_{l}$ and receiver $\mathrm{RX}_{k}$ are drawn independently from the same distribution $h_{l k} \sim$ $\mathcal{C N}\left(0, \sigma_{I}^{2}\right)$, while the desired channel gains $h_{k k}$ between transmitter $\mathrm{TX}_{k}$ and receiver $\mathrm{RX}_{k}$ are drawn independently from the distribution $h_{k k} \sim \mathcal{C N}\left(0, \sigma_{S}^{2}\right)$. Hence, the parameters of our system are $K, \sigma_{I}^{2}$ and $\sigma_{S}^{2}$.

\section{B. Discrete Superposition Model}

The DSM of the $K$-CIFC has the same channel gains $h_{l k}$, however, the relation between transmit and receive signals is different. The value of the transmit symbols $x_{k}$ are chosen from a set of discrete values as follows:

$$
x_{k} \in \mathcal{D}+j \mathcal{D}
$$

where $\mathcal{D}$ is the set of equidistant values defined as follows:

$$
\mathcal{D}=\left\{0, \frac{2^{-n}}{\sqrt{2}}, \ldots, \frac{1-2^{-n}}{\sqrt{2}}\right\} .
$$

Hence, there are $2^{2 n}$ possible transmit symbols at each transmitter where $n$ is equal to:

$$
n=\max _{(l, k)} \max \left\{\left\lfloor\log \left|h_{l k \mathrm{R}}\right|\right\rfloor,\left\lfloor\log \left|h_{l k \mathrm{I}}\right|\right\rfloor\right\} .
$$

The first maximum in (4) is over all links in the $K$-CIFC, and the indices $\mathrm{R}$ and $\mathrm{I}$ denote real and imaginary part, respectively. Note that all logarithms in this papers are to the base 2 .

The receive signal at receiver $\mathrm{RX}_{k}$ is

$$
y_{k}=\sum_{l=1}^{K}\left[\left[h_{l k}\right] x_{l}\right]
$$

where $[\cdot]$ is a rounding operation defined as

$$
[a]=\operatorname{sign}\left(a_{\mathrm{R}}\right)\left\lfloor\left|a_{\mathrm{R}}\right|\right\rfloor+j \operatorname{sign}\left(a_{\mathrm{I}}\right)\left\lfloor\left|a_{\mathrm{I}}\right|\right\rfloor .
$$

In general, it is easier to analyze the capacity region of the DSM, which is mainly due to the lack of stochastic noise. Studying the DSM capacity region is reasonable, because of the following relation for the $K$-user interference channel ( $K$ IFC), that is, the $K$-CIFC without message knowledge at the transmitters.

Theorem 1. [10, Theorem 4.2]. The capacity regions of the K-IFC in the AWGN model and the DSM are within a constant gap. That is, if $\left(R_{k}^{\mathrm{D}}\right)$ is a rate tuple in the capacity region of the DSM K-IFC, then there exists a rate tuple $\left(R_{k}^{\mathrm{G}}\right)$ in the corresponding AWGN K-IFC with

$$
R_{k}^{\mathrm{G}} \geq R_{k}^{\mathrm{D}}-\kappa_{1}, \quad k \in\{1, \ldots, K\} .
$$

Conversely, if $\left(R_{k}^{\mathrm{G}}\right)$ is a rate tuple in the capacity region of the AWGN K-IFC, then there exists a rate tuple $\left(R_{k}^{\mathrm{D}}\right)$ in the capacity region of the corresponding DSM K-IFC with

$$
R_{k}^{\mathrm{D}} \geq R_{k}^{\mathrm{G}}-\kappa_{2}, \quad k \in\{1, \ldots, K\} .
$$

The constants $\kappa_{1}$ and $\kappa_{2}$ are independent of the channel gains.

Specifically, there exists a strategy to "lift" any code from the DSM to a code in the AWGN model. The rate tuples of the codes satisfy (7). The lifting procedure also works for the $K$-CIFC, because it is irrespective of the message knowledge. This yields the following corollary.

Corollary 1. If $\left(R_{k}^{\mathrm{D}}\right)$ is a rate tuple in the capacity region of the DSM K-CIFC, then there exists a rate tuple $\left(R_{k}^{\mathrm{G}}\right)$ in the capacity region of the corresponding $A W G N K$-CIFC with

$$
R_{k}^{\mathrm{G}} \geq R_{k}^{\mathrm{D}}-\kappa_{1}, \quad k \in\{1, \ldots, K\} .
$$

The constant $\kappa_{1}$ is independent of the channel gains.

Note that the converse of Theorem 1 does not immediately carry over to the case of $K$-CIFC. Since we consider achievable rates in this paper, we do not require the converse.

\section{Achievable Rate Tuple in Discrete SUPERPOSITION MODEL}

In this section we state our first result, the tuple of achievable rates in the DSM.

Theorem 2. On the K-CIFC in the DSM we can achieve the rate tuple

$$
\begin{aligned}
& R_{1}=\log \left|h_{11}\right|^{2}-10, \\
& R_{k}=\log \left|h_{k k}\right|^{2}-\left(\max _{l \neq k} \log \left|h_{k l}\right|^{2}\right)^{+}-11, k \in\{2, \ldots, K\} .
\end{aligned}
$$


Note that all rates have to be positive, that is, if (10) yields a negative value, the corresponding pair cannot operate. The result can be obtained from extending a result for the CIFC with a single secondary pair [12]. The transmission strategy is based on two main ideas. Firstly, the secondary transmitters avoid causing interference at the primary receiver and all undesired secondary receivers, that is,

$\left[\left[h_{k l}\right] x_{k}\right]=0$, for all $l \in\{1, \ldots, K\} \backslash l$ and $k \in\{2, \ldots, K\}$.

Secondly, since the secondary transmitters know the primary message, they can pre-cancel the interference caused by the primary transmitter $\mathrm{TX}_{1}$ by using a simple version of DPC.

Let us consider the primary system. The primary receiver does not receive any interference. Hence, the received signal is

$$
y_{1}=\left[\left[h_{11}\right] x_{1}\right],
$$

and the rate $R_{1}$ is only limited by the direct channel gain $h_{11}$. Recall that the transmit signal $x_{1}$ has discrete values according to (2). Some of these values for $x_{1}$ merge to the same value for $y_{1}$ due to the rounding operation in (12). The transmitter compensates this by using only a subset of the possible values for $x_{1}$, which guarantees error free reception at the receiver. The resulting rate (10a) can be obtained from a worst-case analysis. The details can be found in [12].

The secondary systems experience two difficulties in their operation, which are interference from the primary transmitter and the requirement to avoid interference at any other receivers. Avoiding interference is achieved by reducing the set of values of the transmit signal $x_{k}$ in order to guarantee (11). Clearly, the maximum cross-channel gain

$$
\left|h_{k, \max }\right|=\max _{l \neq k}\left|h_{k l}\right|
$$

is the limiting parameter for the set of possible transmit values. From

$$
\left[\left[h_{k, \max }\right] x_{k}\right]=0
$$

we find the region of permissible values for $x_{k}$. This causes the second term in (10b). The interference from the primary transmitter can be effectively compressed by using DPC implemented by lattice coding based on a two dimensional lattice. The received signal at receiver $\mathrm{RX}_{k}$ is

$$
y_{k}=\left[\left[h_{k k}\right] x_{k}\right]+\left[\left[h_{1 k}\right] x_{1}\right] .
$$

Assume that the receiver $\mathrm{RX}_{k}$ quantizes $y_{k}$ with respect to a square lattice. The result of the quantization is

$$
\tilde{y}_{k}=y_{k \mathrm{R}} \bmod L+j y_{k \mathrm{I}} \bmod L,
$$

where mod denotes the modulo operation, and $L$ is the size of the square lattice. Since the transmitter $\mathrm{TX}_{k}$ knows the interfering signal $x_{1}$ and the lattice size $L$, it can infer the result of the quantization $\tilde{y}_{k}$. By selecting its own transmit signal $x_{k}$, it can, thus, compensate the effect of interference. Note that this strategy works irrespective of the strength $\left|h_{1 k}\right|$ of the interference, which is a characteristic feature of DPC.
By analyzing the worst case scenario we can find a lower bound on $L$, which results in a lower bound on the number of possible transmit values for $x_{k}$. Together with the constraints due to interference avoidance we find the achievable rate (10b). Details of the calculations can be found in [12].

Note that the secondary rate $R_{k}, k \geq 2$ is positive only if the maximum interfering cross-link $h_{k, \max }$ is larger than the direct link $h_{k k}$. Hence, our scheme is suitable only in the weak interference regime due to the strategy of interference avoidance.

\section{Average Throughrut}

In this section we would like to calculate the average achievable rate $\bar{R}_{k}=\mathrm{E}\left[R_{k}\right]$ of the secondary transmitterreceiver pairs. We find the following result.

Theorem 3. For i.i.d. channel gains $h_{k k} \sim \mathcal{C N}\left(0, \sigma_{S}^{2}\right)$ and $h_{k l} \sim \mathcal{C N}\left(0, \sigma_{I}^{2}\right)$ we obtain

$$
\begin{aligned}
\bar{R}^{\prime} & =\mathrm{E}\left[\log \left|h_{k k}\right|^{2}-\max _{l \neq k} \log \left|h_{k l}\right|^{2}\right] \\
& =(K-1) \sum_{l=0}^{K-2} \frac{(-1)^{l}}{l+1}\left(\begin{array}{c}
K-2 \\
l
\end{array}\right) \log \left(1+(l+1) \frac{\sigma_{S}^{2}}{\sigma_{I}^{2}}\right) .
\end{aligned}
$$

The calculation can be found in Appendix A. Note that Theorem 3 gives the expectation of

$$
R_{k}^{\prime}=\log \left|h_{k k}\right|^{2}-\max _{l \neq k} \log \left|h_{k l}\right|^{2}
$$

without the limiting operation $(\cdot)^{+}$. The expectation of $R_{k}$ is difficult to obtain, however, for high SNR and large $K$, $R_{k}=R_{k}^{\prime}-11$ with high probability, because the maximum is likely to be greater than zero. We confirm this by numerical simulation.

\section{Performance Evaluation}

In this section we evaluate Theorem 3 numerically. To facilitate interpretation, we use the parameters

$$
\mathrm{SNR}=\sigma_{S}^{2}, \quad \alpha=\frac{\log \sigma_{I}}{\log \sigma_{S}},
$$

that is, $\sigma_{I}=\sigma_{S}^{\alpha}$. The use of the interference exponent $\alpha$ is common in the study of the generalized degree of freedom (DOF) [13].

Fig. 2 shows the average secondary throughput $\bar{R}^{\prime}$ as a function of SNR and $K$. Firstly, we check the influence of the $(.)^{+}$operation. The solid lines depict our closed-form expression (17), while the dashed lines result from simulating (10b) through Monte Carlo simulation. We observe that for $K=2$ the approximation is justified only for high values of SNR. However, for larger $K$ the approximation is valid for all values of SNR. The throughput increases with SNR, approaching a linear behavior. It decreases with $K$, however, the degradation is mild and saturates for large $K$. Fig. 3 shows the dependence on the interference exponent $\alpha$ for $K=4$. The smaller $\alpha$, that is, the smaller the interfering cross-links, the larger is the slope of the curves. 


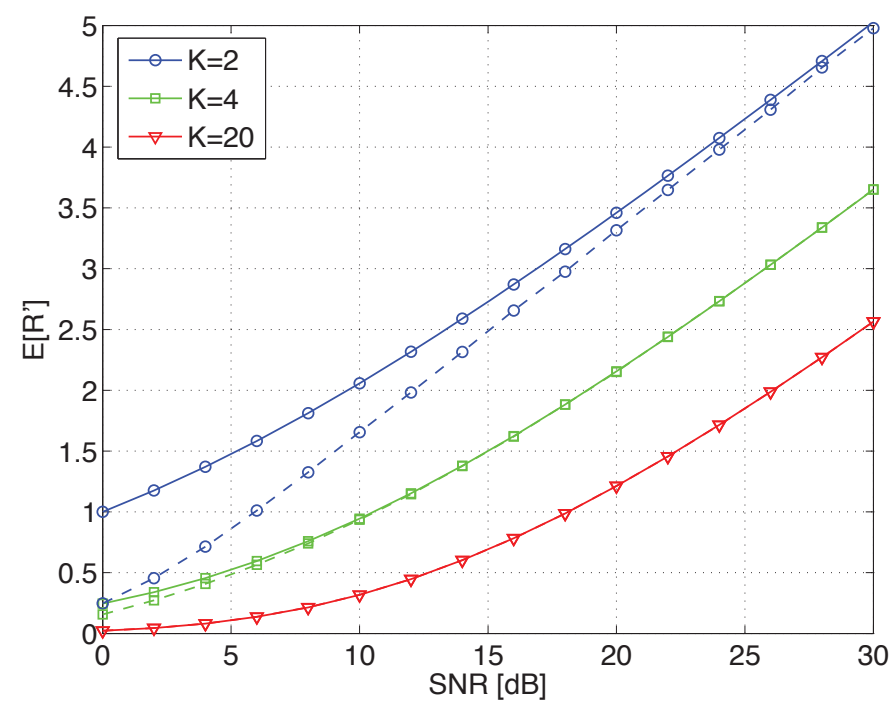

Fig. 2. Average secondary throughput $\bar{R}^{\prime}$ as a function of SNR and number $K$ of users (lines). The interference exponent is $\alpha=0.5$. Dashed lines denote results from numerical simulations

Finally, we show the dependence on $K$ in Fig. 4 and Fig. 5. Fig. 4 shows the secondary throughput $\bar{R}^{\prime}$ as a function of $K$ for different values of SNR and $\alpha=0.5$. The throughput drops steeply for low $K$ due to the increased requirements on interference avoidance. However, for large $K$ the decrease of throughput is small. The throughput stabilizes at a constant non-zero level. This behavior results in an almost linear increase of the sum throughput $(K-1) \bar{R}^{\prime}$ as shown in Fig. 4 for the same set of parameters. The slope of the curve increases with increasing SNR. This last result shows that operating multiple secondary transmitter-receiver pairs is beneficial under the assumptions of this work.

\section{CONCLUSION}

In this paper we found a tuple of achievable rates for the cognitive interference channel with multiple secondary transmitter-receiver pairs. The coding scheme was obtained in the discrete superposition model and yields a coding scheme in the corresponding AWGN model. Furthermore, we found a closed-form expression for the expected secondary throughput for Rayleigh-fading channel gains. We investigated the result numerically and discussed the dependence on the SNR, the interference exponent $\alpha$ and the number of users $K$. Even though the individual throughput decreases with $K$, the sum throughput increases almost linearly. This shows that for our scenario the coexistence and simultaneous operation of multiple secondary systems is beneficial from a sum throughput point of view.

\section{APPENDIX A \\ PROOF OF THEOREM 3}

To calculate the average, the distribution of the random variable $R_{k}^{\prime}$ should be found. The desired channel $h_{k k}$ and the interference channel $h_{k l}$ gains have complex Gaussian distribution with different variances $h_{k k} \sim \mathcal{C N}\left(0, \sigma_{S}^{2}\right), h_{k l} \sim$

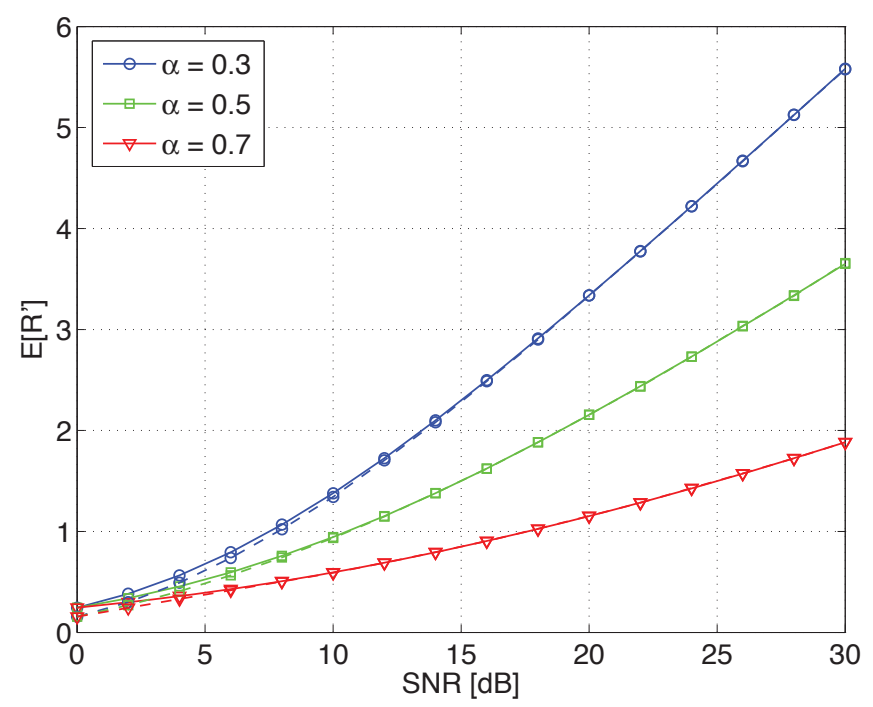

Fig. 3. Average secondary throughput $\bar{R}^{\prime}$ as a function of SNR and interference exponent $\alpha$ (lines). The number of users is $K=4$. Dashed lines results from numerical simulations.

$\mathcal{C N}\left(0, \sigma_{I}^{2}\right)$. The squared magnitudes of the channel gains have exponential distributions as follows,

$$
\begin{gathered}
X_{k}=\left|h_{k k}\right|^{2} \sim f_{X}(x)=\frac{1}{\sigma_{S}^{2}} e^{-\frac{x}{\sigma_{S}^{2}}}, x \geq 0, \\
W_{k l}=\left|h_{k l}\right|^{2} \sim f_{W}(w)=\frac{1}{\sigma_{I}^{2}} e^{-\frac{w}{\sigma_{I}^{2}}}, w \geq 0 .
\end{gathered}
$$

Now, we define a new random variable $Y_{k}$ as follows,

$$
Y_{k}=\max _{l=1, \ldots, K, l \neq k} W_{k l} \text {. }
$$

The cumulative probability distribution of $Y_{k}$ can be found as

$$
\begin{aligned}
F_{Y}(y) & =\operatorname{Pr}\left\{Y_{k} \leq y\right\}=\prod_{l=1, l \neq k}^{K} \operatorname{Pr}\left\{W_{l k} \leq y\right\} \\
& =\left(1-e^{-\frac{y}{\sigma_{I}^{2}}}\right)^{K-1} .
\end{aligned}
$$

The resulting probability density function of $Y_{k}$ is

$$
f_{Y}(y)=\frac{d}{d y} F_{Y}(y)=\frac{1}{\sigma_{I}^{2}}(K-1) e^{-\frac{y}{\sigma_{I}^{2}}}\left(1-e^{-\frac{y}{\sigma_{I}^{2}}}\right)^{K-2}
$$

The probability density function of the fraction $Z_{k}=\frac{X_{k}}{Y_{k}}$ is

$$
\begin{aligned}
f_{Z}(z)= & \int_{-\infty}^{\infty}|y| f_{X, Y}(z y, y) d y \\
= & \int_{0}^{\infty} y f_{X}(z y) f_{Y}(y) d y \\
= & \frac{K-1}{\sigma_{S}^{2} \sigma_{I}^{2}} \\
& \times \int_{0}^{\infty} y e^{-\left(\frac{z}{\sigma_{S}^{2}}+\frac{1}{\sigma_{I}^{2}}\right) y}\left(1-e^{\frac{-y}{\sigma_{I}^{2}}}\right)^{K-2} d y .
\end{aligned}
$$




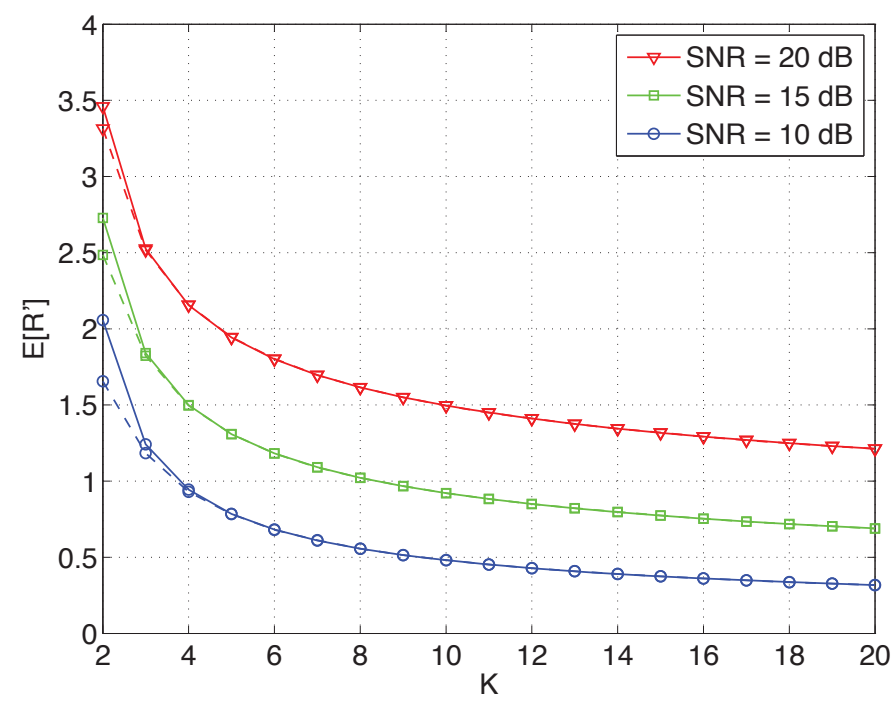

Fig. 4. Average secondary throughput $\bar{R}_{k}^{\prime}$ as a function of number $K$ of users and SNR (lines). The interference exponent is $\alpha=0.5$. Dashed lines denote results from numerical simulations.

To solve the integral, we observe

$$
\begin{aligned}
\int_{0}^{\infty} x e^{-a x}\left(1-e^{-b x}\right)^{K} d x \\
\quad=\int_{0}^{\infty} x e^{-a x} \sum_{l=0}^{K}\left(\begin{array}{c}
K \\
l
\end{array}\right)(-1)^{l} e^{-b l x} \\
=\sum_{l=0}^{K}\left(\begin{array}{c}
K \\
l
\end{array}\right)(-1)^{l} \int_{0}^{\infty} x e^{-(a+b l) x} d x \\
=\sum_{l=0}^{K}\left(\begin{array}{c}
K \\
l
\end{array}\right) \frac{(-1)^{l}}{(a+b l)^{2}} .
\end{aligned}
$$

This yields

$$
\begin{aligned}
f_{Z}(z)= & \frac{\sigma_{S}^{2}(K-1)}{\sigma_{I}^{2}} \\
& \times \sum_{l=0}^{K-2}\left(\begin{array}{c}
K-2 \\
l
\end{array}\right) \frac{-1^{l}}{\left(z+\frac{\sigma_{S}^{2}}{\sigma_{I}^{2}}(l+1)\right)^{2}} .
\end{aligned}
$$

We calculate the expectation of the logarithm of the fraction $Z_{k}$

$$
\begin{aligned}
\mathrm{E}[ & \left.\log Z_{k}\right] \\
= & \int_{1}^{\infty} f_{Z}(z) \log z d z \\
= & (K-1) \frac{\sigma_{S}^{2}}{\sigma_{I}^{2}} \\
& \times \sum_{l=0}^{K-2}\left(\begin{array}{c}
K-2 \\
l
\end{array}\right)(-1)^{l} \int_{1}^{\infty} \frac{\log z}{\left(z+(l+1) \frac{\sigma_{S}^{2}}{\sigma_{I}^{2}}\right)^{2}} d z \\
= & (K-1) \\
& \times \sum_{l=0}^{K-2}\left(\begin{array}{c}
K-2 \\
l
\end{array}\right) \frac{(-1)^{l}}{l+1} \log \left(1+\frac{\sigma_{S}^{2}}{\sigma_{I}^{2}}(l+1)\right),
\end{aligned}
$$

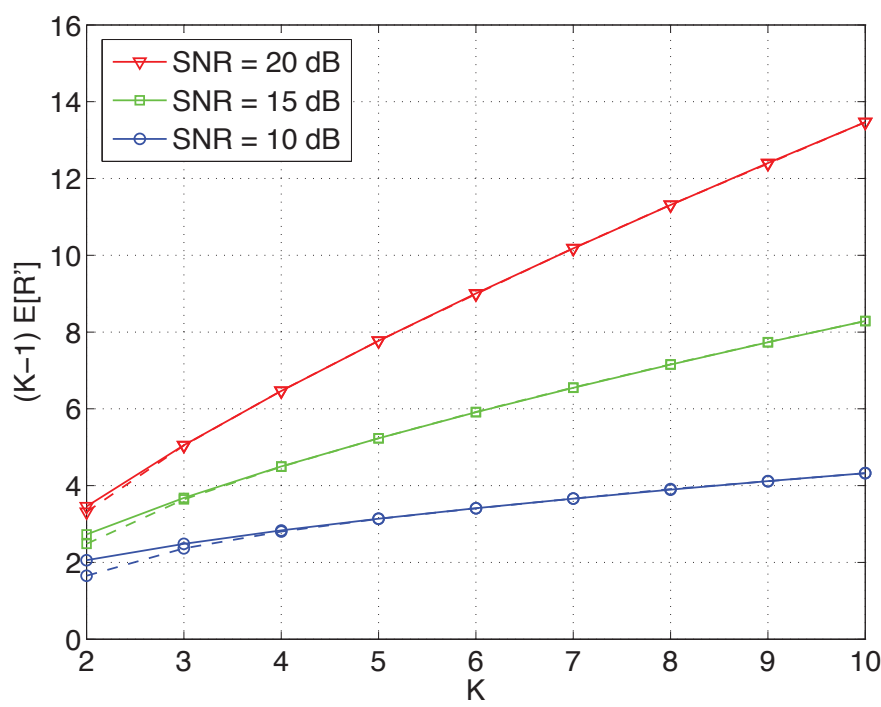

Fig. 5. Average secondary sum throughput $(K-1) \bar{R}_{k}^{\prime}$ as a function of number $K$ of users and SNR (lines). The interference exponent is $\alpha=0.5$. Dashed lines denote results from numerical simulations.

which yields the expected throughput $\bar{R}^{\prime}$.

\section{REFERENCES}

[1] J. Mitola III and G. Maguire Jr., "Cognitive radio: making software radios more personal," IEEE Pers. Comm., vol. 6, no. 4, pp. 13-18, Aug. 1999.

[2] N. Devroye, P. Mitran, and V. Tarokh, "Achievable rates in cognitive radio channels," IEEE Trans. Inf. Theory, vol. 52, no. 5, pp. 1813-1827, May 2006.

[3] W. Wu, S. Vishwanath, and A. Arapostathis, "Capacity of a class of cognitive radio channels: Interference channels with degraded message sets," IEEE Trans. Inf. Theory, vol. 53, pp. 4391-4399, May 2007.

[4] I. Maric, R. Yates, and G. Kramer, "Capacity of interference channels with partial transmitter cooperation," IEEE Trans. Inf. Theory, vol. 53, no. 10 , pp. 3536-3548, Oct. 2007.

[5] S. Rini, D. Tuninetti, and N. Devroye, "New results on the capacity of the Gaussian cognitive interference channel," in Proc. Allerton Conf. Commun., Control and Comp., Sep. 2010.

[6] _ - "Inner and outer bounds for the Gaussian cognitive interference channel and new capacity results," submitted to IEEE Trans. Inf. Theory, online arXiv: 1010.5806.

[7] A. Jafarian and Vish, "On the capacity of multi-user cognitive radio networks," in Proc. Int. Symp. Inf. Theory (ISIT), 2009.

[8] K. Nagananda and C. Murthy, "Three-user cognitive channels with cumulative message sharing: An achievable rate region," in Proc. Inf. Theory Worksh. (ITW), Jun. 2009, pp. $291-295$.

[9] A. Goldsmith, S. Jafar, I. Maric, and S. Srinivasa, "Breaking spectrum gridlock with cognitive radios: An information theoretic perspective," Proc. of IEEE, vol. 97, no. 5, pp. 894 - 914, May 2009.

[10] M. Anand and P. Kumar, "A digital interface for Gaussian relay and interference networks: Lifting codes from the discrete superposition model," IEEE Trans. Inf. Theory, vol. 57, pp. 2548-2564, May 2011.

[11] M. Costa, "Writing on dirty paper," IEEE Trans. Inf. Theory, vol. 29, no. 3, pp. 439-441, May 1983.

[12] N. Schrammar, Ed., Detection Performance of Cooperative Schemes. QUASAR Deliverable D2.3, Oct. 2011, online: www.quasarspectrum.eu.

[13] R. Etkin, D. Tse, and H. Wang, "Gaussian interference channel capacity to within one bit," IEEE Trans. Inf. Theory, vol. 54, no. 12, pp. 5534 5562, 2008. 